International Journal of Multidisciplinary Research AND ANALysis

ISSN(print): 2643-9840, ISSN(online): 2643-9875

Volume 04 Issue 08 August 2021

DOI: 10.47191/ijmra/v4-i8-03, Impact Factor: 6.072

Page No.- 1072-1075

\title{
Evaluation of Pelvic Floor Dysfunction for Patient with History of Total Vaginal Hysterectomy at Dr Sardjito General Hospital Yogyakarta
}

\author{
Mochammad Andi Gunawan ${ }^{1}$, Nuring Pangastuti ${ }^{2}$ \\ ${ }^{1,2}$ Department of Obstetrics and Gynaecology, Dr. Sardjito Hospital-Faculty of Medicine, Public Health and Nursing, Universitas \\ Gadjah Mada, Indonesia
}

\begin{abstract}
:
Introduction: Uterine prolapse is the herniation of the uterus into or beyond the vagina as a result of failure of the ligamentous and fascial supports. It often coexist with prolapse of the vaginal walls, involving the bladder or rectum. A surgical treatment for uterine prolapse is hysterectomy. Changes in pelvic anatomic structure due to hysterectomy can result in impaired pelvic function because of nerve or pelvic muscle structure damage during surgery.
\end{abstract}

Objective: This study aims to evaluate the presence of pelvic floor dysfunction in uterine prolapse patients who have undergone Total Vaginal Hysterectomy (TVH) surgery at Dr Sardjito General Hospital, from November 2018 to September 2019. Evaluations were carried out using Pelvic Floor Distress Inventory (PFDI) questionnaire.

Methods: This study is an analytical descriptive research, using secondary data taken from patient's medical records at Dr. Sardjito General Hospital Yogyakarta. It uses The Pelvic Floor Distress Inventory (PFDI) questionnaire, which consisted of 3 assessment groups, those are Pelvic Organ Prolapse Distress Inventory 6 (POPDI-6), Colorectal-Anal Distress Inventory 8m(CRADI 8), and Urinary Distress Inventory 6 (UDI- 6). The inclusion criteria of this study were patients with diagnoses of uterine prolapse, cystocele and rectocele, who could be contacted and gave consent to conduct telephone interview.

Results: There were 14 patients that met the inclusion criteria, they are uterine prolapse, cystocele and rectocele patients with a history of TVH procedure, were assessed using PFDI questionnaire. All patients did not have any pelvic floor dysfunction either from the POPDI-6, CRADI-8 or UDI-6 assessment groups. The weakness of this evaluation is that the PFDI postoperative value cannot be compared with before surgery, so it cannot be proven that the absence of pelvic floor dysfunction in the patient concerned is a positive result of the surgery performed, or not give significant changes.

Conclusion: There is no pelvic floor dysfunction in patients with a history of TVH procedure on the indication of uterine prolapse, which was performed at Dr Sardjito General Hospital Yogyakarta.

KEY WORDS: Hysterectomy, Total Vaginal Hysterectomy, uterine prolapse, PFDI

\section{INTRODUCTION}

Uterine prolapse is the herniation of the uterus into or beyond the vagina as a result of failure of the ligamentous and fascial supports. It often coexist with prolapse of the vaginal walls, involving the bladder or rectum. ${ }^{1}$

A surgical treatment for uterine prolapse is hysterectomy. Hysterectomy for uterine prolapse can be performed via the abdominal route of vaginal route (total vaginal hysterectomy). ${ }^{1}$ Total vaginal hysterectomy has fewer complication, requires a shorter hospital stay, and allows a faster recovery compared with removal of the uterus through an abdominal incision (abdominal hysterectomy. ${ }^{2}$ Total Vaginal Hysterectomy (TVH) procedure is a surgical procedure to remove the entire uterus, including the cervix. This procedure is often performed in gynaecological surgery.

A number of complication can occurs of total vaginal hysterectomy is haemorrhage occurs in a small number of cases, urinary disorders, defecation disorders, sexual function disorders, damage to adjacent organs, and early menopause. ${ }^{3}$ 


\section{Evaluation of Pelvic Floor Dysfunction for Patient with History of Total Vaginal Hysterectomy at Dr Sardjito General Hospital Yogyakarta}

Changes in pelvic anatomic structure due to hysterectomy can result in impaired pelvic function because of nerve or pelvic muscle structure damage during surgery. In Dr Sardjito General Hospital Yogyakarta, TVH procedure was mostly done to a patient with uterine prolapse indication and it was necessary to evaluate their pelvic floor function after the procedure.

\section{Objective}

This study aims to evaluate the presence of pelvic floor dysfunction in uterine prolapse patients who have undergone Total Vaginal Hysterectomy (TVH) surgery at Dr Sardjito General Hospital, from November 2018 to September 2019. Evaluations were carried out using Pelvic Floor Distress Inventory (PFDI) questionnaire.

\section{SUBJECTS AND METHODS}

This study used a subject with uterine prolapse who had performed a total vaginal hysterectomy at Dr. Sardjto General Hospital from November 2018 to September 2019.

This study is an analytical descriptive research, using secondary data taken from patient's medical records at Dr. Sardjito General Hospital Yogyakarta. It uses The Pelvic Floor Distress Inventory (PFDI) questionnaire, which consisted of 3 assessment groups, those are Pelvic Organ Prolapse Distress Inventory 6 (POPDI-6) (Table 1), Colorectal-Anal Distress Inventory 8 (CRADI 8) (Table 2), and Urinary Distress Inventory 6 (UDI- 6) (Table 3). All items have a response scale from 0 to 4 . Symptoms presents $=$ YES, scale of bother ( 1 : not at all, 2 : somewhat, $3:$ moderately, $4:$ quite a bit) and symptoms not presents $=$ NO $(0)$.

The Pelvic Floor Distress Inventory (PFDI) questionnaire have a questions about bowel, bladder, or pelvic symptoms and how much they bother. Symptoms are asked for 3 months after total vaginal hysterectomy.

The inclusion criteria of this study were patients with diagnoses of uterine prolapse, cystocele and rectocele, who could be contacted and gave consent to conduct telephone interview.

Scoring the PFDI is Obtain the mean value of all of the answered items within the corresponding scale (possible value 0 to 4 ) and then multiply by 25 to obtain the scale score (range 0 to 100) and add the score from the 3 scales together to obtain the summary score (range 0 until 100)

Table 1. Pelvic Organ Prolapse Distress Inventory (POPDI) - PFDI

\section{Pelvic Organ Prolapse Distress Inventory 6 (POPDI-6)}

\begin{tabular}{|c|c|c|}
\hline Do you ... & No & Yes \\
\hline 1. Usually experience pressure in the lower abdomen? & 0 & 123 \\
\hline 2. Usually experience heaviness or dullness in the pelvic area? & 0 & 12 \\
\hline $\begin{array}{l}\text { 3. Usually have a bulge or something falling out that you can see or feel in your vaginal } \\
\text { area? }\end{array}$ & 0 & 12 \\
\hline $\begin{array}{l}\text { 4. Ever have to push on the vagina or around the rectum to have or complete a bowel } \\
\text { movement? }\end{array}$ & 0 & 12 \\
\hline 5. Usually experience a feeling of incomplete bladder emptying? & 0 & 12 \\
\hline $\begin{array}{l}\text { 6. Ever have to push up on a bulge in the vaginal area with your fingers to start or complete } \\
\text { urination? }\end{array}$ & 0 & 12 \\
\hline
\end{tabular}

Table 2. Colorectal Organ Distress Inventory (CRAD) - PFDI

\section{Urinary Distress Inventory 6 (UDI-6):}

\begin{tabular}{|c|c|c|}
\hline Do you ... & No & Yes \\
\hline 15. Usually experience frequent urination? & 0 & $\begin{array}{llll}1 & 2 & 3 & 4\end{array}$ \\
\hline $\begin{array}{l}\text { 16. Usually experience urine leakage associated with a feeling of urgency, that is, a strong } \\
\text { sensation of needing to go to the bathroom? }\end{array}$ & 0 & 12 \\
\hline 17. Usually experience urine leakage related to coughing, sneezing, or laughing? & 0 & 34 \\
\hline 18. Usually experience small amounts of urine leakage (that is, drops)? & 0 & 12 \\
\hline 19. Usually experience difficulty emptying your bladder? & 0 & 12 \\
\hline 20. Usually experience pain or discomfort in the lower abdomen or genital region? & 0 & 12 \\
\hline
\end{tabular}




\section{Evaluation of Pelvic Floor Dysfunction for Patient with History of Total Vaginal Hysterectomy at Dr Sardjito General Hospital Yogyakarta}

Table 3. Urinary Distress Inventory (UDI) - PFDI

\section{Colorectal-Anal Distress Inventory 8 (CRAD-8):}

\begin{tabular}{|c|c|c|}
\hline Do уои ... & No & Yes \\
\hline 7. Feel you need to strain too hard to have a bowel movement? & 0 & 123 \\
\hline 8. Feel you have not completely emptied your bowels at the end of a bowel movement? & 0 & 2 \\
\hline 9. Usually lose stool beyond your control if your stool is well formed? & 0 & 3 \\
\hline 10. Usually lose stool beyond your control if your stool is loose? & 0 & 2 \\
\hline 11. Usually lose gas from the rectum beyond your control? & 0 & 2 \\
\hline 12. Usually have pain when you pass your stool? & 0 & 2 \\
\hline $\begin{array}{l}\text { 13. Experience a strong sense of urgency and have to rush to the bathroom to have a bowel } \\
\text { movement? }\end{array}$ & 0 & 12 \\
\hline $\begin{array}{l}\text { 14. Does part of your bowel ever pass through the rectum and bulge outside during or after a } \\
\text { bowel movement? }\end{array}$ & 0 & 2 \\
\hline
\end{tabular}

\section{RESULTS}

There were 14 patients that met the inclusion criteria, they are uterine prolapse, cystocele and rectocele patients with a history of TVH procedure, were assessed using PFDI questionnaire. All patients did not have any pelvic floor dysfunction either from the POPDI-6, CRADI-8 or UDI-6 assessment groups. PFDI summary score is 0 (symptoms not presents)

\section{DISCUSSION}

Total vagina hysterectomy is one of the most commonly performed gynaecological operation on uterine prolapse. The main goal of hysterectomy is to resolve these symptoms in order to improve quality of life. In s some patients this goal is not realized due to unwanted side effects related to the procedure itself.

The prevalence of micturition symptoms, defecation symptoms and sexual dysfunction is increased in patients who have undergone a hysterectomy. However, the increased prevalence of micturition symptoms, defecation symptoms and sexual dysfunction may have been present before hysterectomy.

Total vaginal hysterectomy can occurs changes in pelvic floor function that are caused from damage to pelvic floor innervation and pelvic fibromuscular structures during surgery.

One of the most common reported adverse effects of hysterectomy are lower urinary tract symptoms (LUTS), particularly urinary incontinence. Development of LUTS could be explained by damage occurring during surgery to the innervation and supportive tissues of the pelvis. Symptoms that more often persisted following vaginal hysterectomy were urgency and obstructive micturition.

Several study have addressed the effect of hysterectomy on the occurrence of constipation due to damage to the pelvis plexus that innervates the colon descended and rectum. However, after hysterectomy can occurs increased rectal sensitivity, is the reverse of autonomic denervation.

According to the secondary data analysis performed on patients with uterine prolapse undergoing the TVH procedure within 11 months (November 2018 - September 2019) it was found that there were 14 patients who underwent the TVH procedure. The following are the results obtained through the secondary data analysis based on medical records at Dr. Sardjito General Hospital. In this study the results of 14 patients were assessed by the PFDI questionnaire. on the POPDI assessment of 14 patients denies complaints pressure in the lower abdomen, heaviness or dullness in the pelvic area, have a bulge or something falling out that you can see or feel in your vaginal area, have to push on the vagina or around the rectum to have or complete a bowel movement, feeling of incomplete bladder emptying, and have to push up on a bulge in the vaginal area with your fingers to start or complete urination. On the CRAD assessment of 14 patients denies complaints need to strain too hard to have a bowel movement, have not completely emptied your bowels at the end of a bowel movement, lose stool beyond your control if your stool is well formed, lose stool beyond your control if your stool is loose, lose gas from the rectum beyond your control, have pain when you pass your stool, strong sense of urgency and have to rush to the bathroom to have a bowel movement, bowel ever pass through the rectum and bulge outside during or after a bowel movement. On the UDI assessment of 14 patients denies complaints frequent urination, urine leakage associated with a feeling of urgency, that is, a strong sensation of needing to go to the bathroom, urine leakage related to coughing, sneezing, or laughing, Small amounts of urine leakage (that is, drops), difficulty emptying your bladder, pain or discomfort in the lower abdomen or genital region. The results POPDI (Pelvic Organ Prolapse Distress Inventory) score 0, CRAD (Colorectal Anal Distress Inventory) score 0 , and UDI (Urinary Distress Inventory) score 0. PFDI summary score 0 (symptoms not presents) 


\section{Evaluation of Pelvic Floor Dysfunction for Patient with History of Total Vaginal Hysterectomy at Dr Sardjito General Hospital Yogyakarta}

The weakness of this evaluation is that the PFDI postoperative value cannot be compared with before surgery, so it cannot be proven that the absence of pelvic floor dysfunction in the patient concerned is a positive result of the surgery performed, or not give significant changes.

\section{CONCLUSION}

There is no pelvic floor dysfunction in patients with a history of TVH procedure on the indication of uterine prolapse, which was performed at Dr Sardjito General Hospital Yogyakarta.

\section{ACKNOWLEDGEMENT}

Paper presented at the 2020 Indonesian Uroginecological Association Annual Scientific Meeting in a poster presentation.

\section{REFERENCES}

1) Anjun Doshani, Roderick E C Teo, Chistopher J Mayne, Douglas G Tincello. Uterine Prolapse. Clinical Review. BMJ 2007; 335 (819).

2) Meeks GR, Harris RL. Surgical approach to hysterectomy: abdominal, laparoscopy-assisted, or vaginal. Clin Obstet Gynecol 1997; 40:886.

3) Harris WJ. Complications of hysterectomy. Clin Obstet Gynecol 1997; 40:928.

4) J.P.W.R Roovers and M.M.E. Lakeman. Effects of Genital Prolapse Srgery and Hysterectomy on Pelvic Floor Function. Facts Views Vision Obgyn. 2009; 1(3): 194-207.

5) Pangastuti N, Santoso Bl, Agustiningsih D, Emilia O. Validation test of Indonesian pelvic floor distress inventory-20 (Indonesian PFDI-20) . J Med Sci, Volume 52, Number 2, 2020 April: 131-137.

http://dx.doi.org/10.19106/JMedSci005202202004. 